Forsch Komplementmed 18 | 6 | 11

\section{Cardiodoron $^{\circledR}$}

\section{Ein Meilenstein in der Integrativen Medizin}

Wenn Stress, Hektik und Nervosität das Leben bestimmen, bleibt dies für Herz und Kreislauf nicht ohne Folgen. «Das gesunde Zusammenspiel unserer vegetativen Rhythmen wird empfindlich gestört», erläutert der Nürnberger Arzt und Naturheilmediziner Dr. Frank Meyer. «Nicht selten ist dabei auch die Kommunikation zwischen Nervensystem und Herzmuskel, zwischen 〈Kopf und Herz〉 betroffen.» Der Organismus reagiert mit einem Symptomenkomplex funktioneller Herzbeschwerden, welche die Lebensqualität des Patienten massiv belasten. Cardiodoron ${ }^{\circledR}$ setzt jedoch nicht nur rein symptomatisch an, sondern wirkt regulierend beziehungsweise rhythmisierend auf das $\mathrm{Zu}$ sammenspiel der vegetativen Rhythmen des
Gesamtorganismus. Von der Atmung über die Verdauung bis zur hormonellen Regulation. Das Präparat stimuliert zudem die rhythmischen Prozesse im Herz-Kreislauf-System und stärkt dieses in seiner ausgleichenden Grundfunktion: Dyskardien und andere stressassoziierte HerzKreislauf-Beschwerden werden deutlich gelindert. Die signifikante Verbesserung der regulativen Prozesse durch Cardiodoron ${ }^{\circledR}$ ist durch wissenschaftliche Studien belegt.

\section{Vielseitige Therapieoptionen}

Cardiodoron ${ }^{\circledR}$ gewinnt in der ärztlichen Praxis zunehmend an Bedeutung als

- Therapeutikum akuter Herz-Kreislauf-Beschwerden bei nicht-organischem Befund,

- langfristige Maßnahme zur effektiven Unterstützung und Wiederherstellung gesundheitsfördernder (salutogener) Prozesse im Organismus, insbesondere im HerzKreislauf-System,

- Begleitung zur Standardtherapie bei der Behandlung bereits manifester Herz-KreislaufErkrankungen.

Darüber hinaus tragen die für Cardiodoron ${ }^{\circledR}$ charakteristischen rhythmisierenden Effekte, wie Dr. Frank Meyer betont, «wesentlich zur Prophylaxe von Herz- und Kreislauferkrankungen (unter anderem Bluthochdruck wie auch koronare Herzerkrankungen) sowie Stoffwechselstörungen (Diabetes mellitus und Folgeerkrankungen) bei.»

Für Fragen und Informationen zur Therapie: WELEDA AG

Medizinisch-wissenschafltiche Abteilung /

Therapieberatung

Tel. +49 7171 919-555

med-wiss@weleda.de

\section{Evidenz zur Phytotherapie bei funk- tionellen Magen-Darm-Erkrankungen erbracht}

Die Phytotherapie steht vor der großen Herausforderung, Beweise für ihre Wirkung liefern zu müssen. «Die Anfordernisse der heutigen Medizin sind, dass man verstehen will, wie ein Medikament funktioniert. Daher stehen pflanzliche Präparate, die vor allem eine empirische Historie haben, heute zunehmend auf dem Prüfstand. Sie müssen beweisen, dass sie Mechanismen bewegen, die tatsächlich in die Krankheit eingreifen», sagte Prof. Dr. Peter Malfertheiner, der diesjährige Präsident der DGVS-Jahrestagung und Chairman des Satelliten-Symposiums. Dass dies auch tatsächlich der Fall ist, zeigen zahlreiche Forschungsergebnisse. Im Rahmen der Jahrestagung der Deutschen Gesellschaft für Verdauungs- und Stoff- wechselkrankheiten (DGVS) stellten nun namhafte Mediziner und Wissenschaftler die Erkenntnisse zur Phytotherapie bei funktionellen Magen-Darm-Erkrankungen vor. Besonders eindrucksvoll waren die Daten einer Iberisamara-Kombination, die belegen, dass dieses Phytopharmakon auf alle wichtigen Ursachen und Symptome funktioneller Magen-Darm-Erkrankungen wirkt, auch bei Sodbrennen in Kombination mit anderen Magenbeschwerden.

Wirknachweis auch auf molekularer Ebene gelungen

«Bei der Phytotherapie existiert immer das Vorurteil, dass es keine richtige wissenschaftliche Evidenz gibt. Ich möchte Sie heute vom Gegenteil überzeugen und darstellen, dass die Phytotherapie viel mehr als ein Mythos ist und dass es tatsächlich definierte molekulare
Targets und Mechanismen gibt», leitete Prof. Dr. Michael Schemann seinen Vortrag ein. In einer bisher unveröffentlichten Untersuchung am Tiermodell gelang es ihm und seinen Kollegen, Phänomene zu entschlüsseln, die sich im neuroviszeralen Bereich nach Gabe von Iberogast abspielen und so die überzeugende Wirkung des Phytopharmakons auf Sodbrennen, Völlegefühl und andere Magenbeschwerden erklären.

\section{Quelle}

Symposium der Steigerwald Arzneimittelwerk $\mathrm{GmbH}$ «Flower Power für den Gastroenterologen» auf der 66. Jahrestagung der Deutschen Gesellschaft für Verdauungsund Stoffwechselkrankheiten am 15.09.2011, Leipzig

Weitere Informationen bei Steigerwald Arzneimittelwerk GmbH

Havelstraße 5, 64295 Darmstadt

Tel. +49 06151 3305-0, Fax -453

\title{
Ticker+++ Ticker+++ Ticker+++ Ticker+++ Ticker+++ Ticker+++ Ticker+++
}

ECIM 2011. Obwohl der Begriff «Integrative Medizin» schon seit knapp 20 Jahren existiert, ringt die medizinische Fachwelt bis heute um eine einheitliche Definition, stellte Prof. Dr. Benno Brinkhaus, Kongresspräsident des diesjährigen 4. Europäischen Kongresses für Integrative Medizin (ECIM) bei der Eröffnung der Veranstaltung fest. Er machte deutlich, dass dies nicht Ausdruck von Unvermögen ist, sondern die Dynamik dieser neuen Medizin wiedergibt, die in Deutschland vielerorts noch in den Kinderschuhen steckt und die Medizin der Zukunft gravierend verändern könnte.

$\mathrm{mMv}$ - multi MED vision -

Berliner Medizinredaktion

mmv@berlin.de
Dr. Loges. Aufgrund der Diskussion um den Einsatz der Hormonersatztherapie bei Wechseljahresbeschwerden suchen betroffene Frauen wie Ärzte nach wirksamen aber sicheren pflanzlichen Arzneimitteln. Einen vielversprechenden Wirkansatz bietet ein Extrakt aus der Wurzel des Sibirischen Rhabarbers (Rheum raponticum) in femi-loges ${ }^{\circledR}$. Der Extrakt überzeugte in wissenschaftlichen Studien durch die effektive Reduktion aller psychischen und neurovegetativen Beschwerden. Umfangreiche Informationen stehen Medizinern im Fachgruppenbereich der neu eingerichteten Themenwebsite www. frauenjahre.info zur Verfügung.

Dr. Loges + Co. GmbH

Med.-wiss. Abteilung

Tel. +49 4171 707-182 oder -118
DZVhÄ. Im November ist das Jahresprogramm Ärztliche Homöopathie 2012 des Deutschen Zentralvereins homöopathischer Ärzte (DZVhÄ) erschienen. Im Zentrum des 116-seitigen Heftes steht die ärztliche Weiter- und Fortbildung. Spezielle Kapitel richten sich an Medizinstudenten, Apotheker, Tierärzte und Zahnärzte. Das Jahresprogramm kann kostenlos in der DZVhÄ-Geschäftsstelle, Am Hofgarten 5, 53113 Bonn, Tel. +49 228 2425-330, Fax -331, E-Mail sekretariat@dzvhae.de bestellt oder von der DZVhÄ-Webseite heruntergeladen werden: www.welt-der-homoeopathie.de.

Christoph Trapp

Pressesprecher des DZVhÄ

presse@dzvhae.de

\section{KARGER}

(1) 2011 S. Karger GmbH, Freiburg 